\title{
Interference with the expression of S1PR1 or STAT3 attenuates valvular damage due to rheumatic heart disease
}

\author{
SHENGLIN XIAN ${ }^{1,2}$, ANG CHEN $^{1,2}$, YUNJIAO WU $^{1,2}$, HONG WEN $^{1,2}$, \\ CHUANGHONG LU ${ }^{1,2}$, FENG HUANG ${ }^{1,2}$ and ZHIYU ZENG ${ }^{1,2}$
}

\author{
${ }^{1}$ Department of Cardiology, The First Affiliated Hospital of Guangxi Medical University; ${ }^{2}$ Guangxi Key Laboratory Base of \\ Precision Medicine in Cardio-Cerebrovascular Diseases Control and Prevention, Guangxi Clinical Research Centre for \\ Cardio-Cerebrovascular Diseases, Guangxi Medical University, Nanning, Guangxi 530021, P.R. China
}

Received October 30, 2020; Accepted June 25, 2021

DOI: $10.3892 /$ ijmm.2021.5012

\begin{abstract}
Rheumatic heart disease (RHD) affects numerous individuals annually; however, its pathogenesis remains unclear. The sphingosine 1-phosphate receptor 1 (S1PR1) and signal transducer and activator of transcription 3 (STAT3) have recently been shown to be involved in valvular damage via the promotion of the differentiation of T helper 17 (Th17) cells during the development of RHD-induced valvular damage. The present study investigated whether altering the expression of S1PR1 or STAT3 attenuates valvular damage due to RHD. Inactivated group A streptococcus (GAS) was used to establish a rat model of RHD. Recombinant adeno-associated viral vectors carrying an S1PR1 overexpression sequence were used to overexpress S1PR1. STAT3 small interfering RNA (STAT3-siRNA) was used to inhibit STAT3 expression. Reverse transcription-quantitative PCR (RT-qPCR) was performed to detect the mRNA expression of S1PR1, STAT3, collagen type III $\alpha 1$ chain (Col3al) and fibroblast-specific protein 1 . Western blotting (WB) and immunohistochemistry were used to detect the levels of S1PR1, STAT3, phosphorylated (p-) STAT3, and retinoic acid-related orphan receptor $\gamma \mathrm{T}(\mathrm{ROR} \gamma \mathrm{t})$
\end{abstract}

Correspondence to: Professor Zhiyu Zeng, Department of Cardiology, The First Affiliated Hospital of Guangxi Medical University, 6 Shuangyong Road, Nanning, Guangxi 530021, P.R. China

E-mail: doctorzyz@126.com

Abbreviations: RHD, rheumatic heart disease; S1PR1, sphingosine 1-phosphate receptor 1; STAT3, signal transducer and activator of transcription 3; Th17, T helper 17; GAS, group A streptococcus; RT-qPCR, reverse transcription-quantitative PCR; Col3a1, collagen type III $\alpha 1$ chain; FSP1, fibroblast-specific protein 1 ; WB, western blotting; p-, phosphorylated; ROR $\gamma$ t, retinoic acid-related orphan receptor $\gamma \mathrm{T}$; ELISAs, enzyme-linked immunosorbent assays; IL, interleukin; H\&E, Hematoxylin and eosin; NS, normal saline; AAV, adeno-associated virus; $t-$, total

Key words: rheumatic heart disease, valvular damage, T helper 17 cell, sphingosine 1-phosphate receptor 1, signal transducer and activator of transcription 3 proteins. Enzyme-linked immunosorbent assays (ELISAs) and immunohistochemistry were used to detect the levels of interleukin (IL)-6 and IL-17. Hematoxylin and eosin (H\&E) staining and Sirius Red staining were performed to evaluate the degree of inflammation and fibrosis in the valvular tissues. S1PR1 expression was decreased in the valvular tissues of the rats with RHD. The levels of IL-6, IL-17 and p-STAT3 in the rats with RHD were increased. The degree of valvular inflammation and fibrosis in the rats with RHD was also increased. The overexpression of S1PR1 and the inhibition of STAT3 reduced the total p-STAT3 level, resulting in decreased levels of IL-6, IL-17 and ROR $\gamma \mathrm{t}$, and a reduced degree of valvular inflammation and fibrosis. These results suggest that the expression of S1PR1 and STAT3 may be involved in valvular tissue damage due to RHD. Thus, strategies designed to interfere with the expression of S1PR1 or STAT3 may affect the expression of Th17 cell-related cytokines and may thus attenuate valvular damage due to RHD.

\section{Introduction}

Rheumatic heart disease (RHD) is a preventable heart disease caused by Streptococcus pyogenes or group A streptococcus (GAS) infection (1). RHD is a leading cause of mortality and disability in young patients, and it remains a serious global public health concern (2). The number of individuals with RHD worldwide exceeds one quarter of the number of individuals with cancer, and the number of associated deaths caused by RHD annually is as high as 250,000 (3). However, studies on RHD continue to examine its pathogenesis, which remains unclear. Precise intervention targets for the prevention or treatment of RHD have not yet been identified, at least to the best of our knowledge. The majority of studies on RHD have focused on the association between its pathogenesis and signalling pathways (4-6). Research on intervention targets that attenuate valvular damage due to RHD is lacking.

Sphingosine 1-phosphate receptor 1 (S1PR1) is a G protein-coupled receptor belonging to the S1PR family. S1PR1 mediates lymphocyte migration, and it is associated with multiple immune (7) and heart diseases (8). S1PR1 primarily plays a role in protecting the heart in patients with heart diseases (9-11), and a high S1PR1 expression 
generally protects the heart during the pathogenesis of heart disease $(9,12)$. Garris et al (13) found that the downregulation of S1PR1 expression increased the levels of phosphorylated (p-) signal transducer and activator of transcription 3 (p-STAT3). A recent study also demonstrated that the S1PR1/STAT3 signalling pathway was involved in the development of valvular damage due to RHD in a rat model, in which S1PR1 expression was downregulated, and the levels of p-STAT3 and $\mathrm{T}$ helper 17 (Th17)-related cytokines were increased (14).

STAT3 is a cellular signal transcription factor that is involved in the regulation of a number of cellular activities (15). STAT3 may regulate the differentiation of $\mathrm{CD}^{+}{ }^{+}$ $\mathrm{T}$ cells into Th17 cells (16). Th17 cells and related cytokines mediate inflammatory and autoimmune responses (17-19). Bas et al (20) demonstrated that the ratio of Th17/Treg cells and the levels of IL-17A were significantly increased in patients with RHD compared with those in subjects in the control group. Similar results were observed in animal models of RHD established in Lewis rats (21). A close association between S1PR1 and STAT3 has been identified, and a number of studies have discussed the role of S1PR1 in the regulation of STAT3 in various diseases (22-25). Therefore, it was hypothesised that the STAT3 pathway is activated during the development of RHD and that it induces $\mathrm{CD}^{+}{ }^{+} \mathrm{T}$ cell differentiation into Th17 cells, and Th17 cell-related inflammatory factors participate in the development of RHD. However, researchers have not yet clearly determined whether this pathway modulates or prevents RHD following intervention. A more important goal is the development of appropriate interventions targeting this pathway to modulate or prevent the occurrence of RHD.

Therefore, the present study interfered with the expression of S1PR1 and STAT3 by overexpressing S1PR1 and inhibiting STAT3 to determine whether these treatments would attenuate RHD-induced valvular damage.

\section{Materials and methods}

The present study aimed to determine whether interfering with the expression of S1PR1 or STAT3 would attenuate RHD-induced valvular damage. A rat model of RHD was established as described in previous studies in order to achieve this goal $(14,26)$.

Antigen preparation. Brain heart infusion fluid medium (Guangdong Huankai Microbial Sci. \& Tech. Co., Ltd.) was used to culture GAS [American Type Culture Collection (ATCC)19615], and the temperature during the cultivation process was maintained at a constant value of $37^{\circ} \mathrm{C}$. After $24 \mathrm{~h}$, GAS was washed with normal saline (NS) and transferred onto $10 \%$ neutral formalin for $12 \mathrm{~h}$ for inactivation. NS was used to wash and resuspend the inactivated GAS, and the concentration was simultaneously adjusted to $4.0 \times 10^{11}$ colony forming units (CFU)/ml (26). The antigen suspension was obtained by emulsifying the suspension via sonication (Sonics \& Materials, Inc.).

In vivo gene therapy. Recombinant adeno-associated virus (AAV; serotype 9) vectors carrying the ratS1PR1 overexpression sequence (S1PR1 overexpression; Hanbio Biotechnology Co., Ltd.) driven by the cTNT promoter were used to overexpress
S1PR1. As the main role of AAV was to carry gene sequences as a vector, it did not affect the experiment itself. Therefore, the AAV control group was injected only with the AAV vector without any sequence to assess whether the AAV vectors altered the results of the experiment. The S1PR1 overexpression sequence is listed in Table I. A rat STAT3 small interfering RNA (siRNA) sequence (5'-GGCTGATCATTTATATAAA-3'; STAT3-siRNA; Hanbio Biotechnology Co., Ltd.) driven by the cTNT promoter in a recombinant AAV vector was used to directly silence STAT3 expression. An AAV control was also used as a negative control to determine whether the AAV vector exerted an effect in the rats.

Immunization of rats. A total of 30 Lewis rats (150-180 g) were purchased from Beijing Vital River Animal Technology Co., Ltd.. All rats were female and weighed 150-180 g at 8 weeks of age. The rats were randomly divided into 5 groups ( $\mathrm{n}=6$ each) for the two parts of the present study. Part I of the study examined the effects of S1PR1 overexpression, and part II examined the effects of STAT3 inhibition. The pathogen-free animal laboratory at the Animal Experiment Centre of Guangxi Medical University provided a satisfactory environment for the rats: The temperature was constantly set to $23^{\circ} \mathrm{C}$, and the fluctuation did not exceed $2^{\circ} \mathrm{C}$; the day/night cycle was $12 \mathrm{~h}$; the movement of the rats in the cage was completely unrestricted; sufficient drinking water and standard rat feed were also provided. All the rats were allowed to adapt to the environment for 5 days prior to the commencement of the experiments. All animal experimental procedures were performed according to the Guidelines for the Ethical Review of Laboratory Animal Welfare of China (GB/T 35892-2018) for the care and use of laboratory animals and were approved by the Medical Ethics Committee of the First Affiliated Hospital of Guangxi Medical University (Approval no. 2019-KY-E-069).

The rats were divided randomly into 5 groups as follows: The control group, AAV control group, RHD group, S1PR1 overexpression group and the STAT3-siRNA group. Each group included 6 rats. The RHD group was the established RHD model. A footpad injection of complete Freund's adjuvant (CFA) (each millilitre contained $1 \mathrm{mg}$ heat-inactivated dry Mycobacterium tuberculosis (H37Ra, ATCC 25177), $0.85 \mathrm{ml}$ paraffin oil and $0.15 \mathrm{ml}$ mannitol mono-oleic acid; cat. no. F5881; Sigma-Aldrich, Merck KGaA) was essential for establishing the rat model of RHD. All rats were maintained on soft bedding to protect their hind feet. In total, 9 weeks were required to establish the rat model of RHD. One hind footpad of each rat was injected initially with $100 \mu \mathrm{l}$ of a solution of inactivated GAS $\left(4.0 \times 10^{11} \mathrm{CFU} / \mathrm{ml}\right)$ and CFA mixed at a ratio of 1:1 (v/v). After 1 week, a subcutaneous injection of $500 \mu 1$ of inactivated GAS $\left(4.0 \times 10^{11} \mathrm{CFU} / \mathrm{ml}\right)$ and CFA mixed at a ratio of $1: 1(\mathrm{v} / \mathrm{v})$ were administered into the abdomen of the rats once weekly at the same interval for 4 weeks. Over the last 4 weeks, a subcutaneous abdominal injection was administered once weekly at the same interval with an adjustment of the injection solution to $500 \mu \mathrm{l}$ of inactivated GAS $\left(4.0 \times 10^{11} \mathrm{CFU} / \mathrm{ml}\right)$. Rats in the S1PR1 overexpression group were injected with $2.5 \times 10^{11}$ viral genome particles once through the tail vein (AAV-S1PR1 overexpression, diluted in $200 \mu 1$ of NS) at the beginning of the experiment. After 3 weeks, the rats received exactly the same treatment as those 
Table I. Sequencing result (S1PR1 overexpression sequence).

Sequence name

S1PR1 overexpression sequence
Sequencing result

S1PR1, sphingosine-1-phosphate receptor 1.

\begin{abstract}
ATGGTGTCCTCCACCAGCATCCCAGTGGTTAAGGCTCTCCGCAGCCAAGTCT CCGACTATGGCAACTATGATATCATAGTCCGGCATTACAACTACACAGGCAAG CTGAACATCGGAGTGGAGAAGGACCATGGCATTAAACTGACTTCAGTGGTG TTCATTCTCATCTGCTGCTTGATCATCCTAGAGAATATATTTGTCTTGCTAACT ATTTGGAAAACCAAGAAGTTCCACCGGCCCATGTACTATTTCATAGGCAACC TAGCCCTCTCGGACCTGTTAGCAGGAGTGGCTTACACAGCTAACCTGCTGTT GTCTGGGGCCACCACCTACAAGCTCACACCTGCCCAGTGGTTTCTGCGGGA AGGAAGTATGTTTGTGGCTCTGTCTGCCTCAGTCTTCAGCCTCCTTGCTATCG CCATTGAGCGCTACATCACCATGCTGAAGATGAAACTACACAACGGCAGCAA CAGCTCGCGCTCCTTTCTGCTGATCAGTGCCTGCTGGGTCATCTCCCTCATCC TGGGTGGGCTGCCCATCATGGGCTGGAACTGCATCAGCTCGCTGTCCAGCTG CTCCACCGTGCTCCCGCTCTACCACAAGCACTATATTCTCTTCTGCACCACCG TCTTCACCCTGCTCCTGCTTTCCATCGTCATCCTCTACTGCAGGATCTACTCC TTGGTGAGGACTCGAAGCCGCCGCCTGACCTTCCGCAAGAACATCTCCAAG GCCAGCCGCAGTTCCGAGAAGTCTCTGGCCTTGCTGAAGACAGTGATCATT GTCCTGAGTGTCTTCATTGCCTGCTGGGCCCCTCTCTTCATCCTACTACTTTT AGATGTGGGGTGCAAGGCGAAGACCTGTGACATCCTGTACAAAGCAGAGTA CTTCCTGGTTCTGGCTGTGCTGAACTCAGGTACCAACCCCATCATCTACACTC TGACCAATAAGGAGATGCGCCGGGCCTTCATCAGGATCATATCTTGTTGCAAA TGCCCCAACGGAGACTCCGCTGGCAAATTCAAGAGGCCCATCATCCCGGGCA TGGAATTTAGCCGCAGCAAATCAGACAACTCCTCCCACCCCCAGAAGGATGA TGGGGACAATCCAGAGACCATTATGTCTTCTGGAAACGTCAATTCTTCT TCT
\end{abstract}

in the RHD group. The rats in the AAV control group received an injection of $2.5 \times 10^{11}$ viral genome particles once through the tail vein (AAV control, diluted with $200 \mu \mathrm{l}$ of NS) at the beginning of the experiment. After 3 weeks, these rats were injected according to the same protocol as that for the RHD group. The rats in the control group were injected using the same protocol as that for the RHD group from the beginning of the experiment, although the injection solution included the same volume of NS. The rats in the STAT3-siRNA group were injected using the same protocol as that for the S1PR1 overexpression group, except that the solution for the tail vein injection was changed from $2.5 \times 10^{11}$ viral genome particles (AAV-S1PR1 overexpression, diluted with $200 \mu \mathrm{l}$ of NS) to $2.5 \times 10^{11}$ viral genome particles (AAV-STAT3-siRNA, diluted with $200 \mu 1$ of NS).

Animal sacrifice. Following the administration of all treatments, $1 \mathrm{ml}$ of blood was collected from the tail vein of the rats in each group without anaesthesia, and an intraperitoneal injection of sodium pentobarbital $(150 \mathrm{mg} / \mathrm{kg})$ was then administered to euthanise the rats. Animal death was determined when $>5$ min had elapsed without breathing or a heartbeat. The humane endpoint in the present study was defined as animals losing $>15 \%$ of their body weight with a decreased ability to consume food and water. None of the rats reached this humane endpoint before the end of the experimental period.

Sample preparation. Valvular samples were collected from each rat. All samples were rapidly frozen in liquid nitrogen and stored at $-80^{\circ} \mathrm{C}$ for use in subsequent experiments. No animals died during the modelling process. The following five experimental methods [reverse transcription-quantitative PCR (RT-qPCR)], western blotting (WB), immunohistochemistry, histochemistry and enzyme-linked immunosorbent assay (ELISA)] were performed on the previously mentioned experimental groups.

$R T-q P C R$. Total RNA was extracted from each sample. TRIzol ${ }^{\circledR}$ reagent (Invitrogen; Thermo Fisher Scientific, Inc.) was used to complete this step according to the manufacturer's protocol. The RNA concentration was measured using a NanoDrop 2000 spectrophotometer (NanoDrop Technologies; Thermo Fisher Scientific, Inc.) for quantitative reverse transcription. RNA was reverse transcribed into cDNA; $0.5 \mu \mathrm{g}$ of total RNA from each sample was reverse transcribed into cDNAs. The PrimeScript RT reagent kit (cat. no. RR036A; Takara Bio, Inc.) was used for reverse transcription. The entire reverse transcription process was performed in accordance with the manufacturer's instructions. RT-qPCR was performed using TB Green Premix Ex Taq II (cat. no. RR820Q; Takara Bio, Inc.), a StepOne system (cat. no. 4376357; Applied Biosystems; Thermo Fisher Scientific, Inc.), and the internal reference gene, $\beta$-actin. The entire process was performed in accordance with the manufacturer's instructions. The thermocycling conditions were as follows: $95^{\circ} \mathrm{C}$ for $30 \mathrm{~min}$, followed by 40 cycles at $95^{\circ} \mathrm{C}$ for $5 \mathrm{sec}$ and $60^{\circ} \mathrm{C}$ for $30 \mathrm{sec}$. The sequences of the primers are listed in Table II. The final results are expressed as the fold change between the expression level of each mRNA and the 
internal reference using the $2^{-\Delta \Delta \mathrm{Cq}}$ method (27). All samples were measured three times.

$W B$. Total protein was extracted from each sample using RIPA lysis buffer (Sangon Biotech Co., Ltd.), according to the manufacturer's instructions. The protein concentration was measured using a bicinchoninic acid (BCA) protein assay kit (Sangon Biotech Co., Ltd.). The same amounts of protein $(30 \mu \mathrm{g})$ from each sample were separated on $10 \%$ SDS-PAGE gels. The separation conditions were $80 \mathrm{~V}$ for $30 \mathrm{~min}$ and $120 \mathrm{~V}$ for $60 \mathrm{~min}$ using a blotting system (Bio-Rad Laboratories, Inc.), according to the manufacturer's instructions. The separated proteins were electrotransferred to $0.22-\mu \mathrm{m}$ polyvinylidene fluoride (PVDF) membranes (EMD Millipore), and the transfer conditions were a constant voltage of $80 \mathrm{~V}$ for $80 \mathrm{~min}$. The membranes were blocked for $1 \mathrm{~h}$ at room temperature in a $3 \%$ bovine serum albumin (BSA) blocking solution (Sangon Biotech Co., Ltd.) and the membranes were then incubated overnight at $4^{\circ} \mathrm{C}$ with the following antibodies: Anti-S1PR1 $(1: 1,000$; cat. no. 55133-1-AP; ProteinTech Group, Inc.), anti-STAT3 (1:1,000; cat. no. ab68153; Abcam), anti-p-STAT3 (1:1,000; cat. no. 9145; Cell Signaling Technology, Inc.) and anti- $\beta$-tubulin $(1: 3,000$; cat. no. 10068-1-AP; ProteinTech Group, Inc.). The membranes were subsequently incubated with an HRP-conjugated secondary antibody (1:10,000; cat. no. ab6721; Abcam) in the dark for $1 \mathrm{~h}$ at room temperature. Protein bands were scanned using a chemiluminescence imaging system (Alpha FluorChem FC3; Alpha, Inc.). The levels of the proteins were normalized to $\beta$-tubulin and quantified using ImageJ software $(1.51 \mathrm{j}$, National Institute of Health). All samples were measured three times.

Histochemistry. The valvular tissues were fixed in $4 \%$ paraformaldehyde for $24 \mathrm{~h}$ at $4^{\circ} \mathrm{C}$ prior to decalcification and embedding in paraffin blocks. All blocks were serially sectioned at a thickness of $5 \mu \mathrm{m}$ for Hematoxylin and eosin (H\&E; cat. no. G1120; Beijing Solarbio Science \& Technology Co., Ltd.) and Sirius Red staining (cat. no. S8060-5; Beijing Solarbio Science \& Technology Co., Ltd.). H\&E staining was performed at room temperature, and the sections were stained with Hematoxylin for 4-10 min followed by eosin for 0.5-2 min. A BX43 light microscope (Olympus Corporation) was used to capture the images of H\&E staining. Sirius Red staining was also performed at room temperature for $1 \mathrm{~h}$. A BX43 confocal microscope (magnification, $\mathrm{x} 400$; Olympus Corporation) was used to capture the images of Sirius Red staining.

Immunohistochemistry. Immunohistochemistry was performed using the method described in a previous study (26) to analyse the valvular tissues stained with antibodies against IL-6 (1:65; cat. no. ab9324; Abcam), IL-17 (1:90; cat. no. ab214588; Abcam), S1PR1 (1:80; cat. no. ab77076; Abcam), STAT3 (1:75; cat. no. ab68153; Abcam), p-STAT3 (1:70; cat. no. ab76315; Abcam) and retinoic acid-related orphan receptor $\gamma \mathrm{T}$ (ROR $\gamma \mathrm{t}$; 1:75; cat. no. 13205-1-AP; ProteinTech Group, Inc.), which is the key transcription factor driving the differentiation of IL-17-producing Th17 cells (28). Briefly, formalin-fixed valvular tissues were embedded in paraffin. All blocks were sectioned at a thickness of $5 \mu \mathrm{m}$. Following
Table II. Sequences of primers used in reverse transcriptionquantitative PCR.

\begin{tabular}{ll} 
Gene & \multicolumn{1}{c}{ Primer sequence $\left(5^{\prime}-3^{\prime}\right)$} \\
\hline STAT3 & F: TTTGAGACAGAGGTGTACCACCAAG \\
& R: ACCACAGGATTGATGCCCAAG \\
S1PR1 & F: GCTTCATCACTCACTACCCTAGCA \\
& R: TTCTCCCTTCCCTCCCTCTC \\
Col3a1 & F: ACTTCTGGTCCTCCTGGTCTGC \\
& R: CGCCTGGCTCACCCTTTCAC \\
FSP1 & F: TGGGGAGAAGGACAGACGAAGC \\
& R: TGGCAATGCAGGACAGGAAGAC \\
3-actin & F: GGAGATTACTGCCCTGGCTCCTA \\
& R: GACTCATCGTACTCCTGCTTGCTG
\end{tabular}

STAT3, signal transducer and activator of transcription 3; S1PR1, sphingosine-1-phosphate receptor 1; Col3a1, collagen type III $\alpha 1$ chain; FSP1, fibroblast-specific protein 1; F, forward; R, reverse.

deparaffinization and rehydration, a 5\% BSA (Beijing Solarbio Science \& Technology Co., Ltd.) solution was used to block the sections at room temperature for $1 \mathrm{~h}$. Following the deactivation of endogenous peroxidases with hydrogen peroxide, the sections were incubated with the primary antibodies described above for $12 \mathrm{~h}$ at $4^{\circ} \mathrm{C}$. A horseradish peroxidase (HRP)-conjugated anti-rabbit (1:10; cat. no. PV-6001; OriGene Technologies, Inc.) or anti-mouse secondary antibody (1:10; cat. no. PV-6002; OriGene Technologies, Inc.) were incubated with the sections for $30 \mathrm{~min}$ at room temperature. After enhanced colour development using diaminobenzidine (DAB), the immunostained tissues were examined under a BX43 light microscope (Olympus Corporation), and positive expression was detected as brownish yellow staining. Quantitative assessment was performed using the methods described by in the study by Friedrichs et al (29). The immunohistochemical score (IHS) is equal to the staining intensity (SI) multiplied by the percentage of positive cells (PP). The SI was scored as follows: 0 points, negative; 1 point, weak; 2 points, moderate; and 3 points, strong. The PP was scored as follows: 0 points, negative; 1 point, $10 \%$ positive cells; 2 points, $11-50 \%$ positive cells; 3 points, $51-80 \%$ positive cells; and 4 points, $>80 \%$ positive cells. The IHS was calculated to describe the results of the quantitative evaluation.

ELISA. ELISA kits (cat. nos. E04640r and E07451r; Cusabio) were used to measure the serum levels of IL-6 and IL-17. The entire process was performed according to the manufacturer's instructions. All samples were measured three times. After the preparation of all reagents, working standards, samples (serum) and assay plates, $100 \mu \mathrm{l}$ of standard solutions and samples were added to each well, covered with an adhesive strip, and incubated at $37^{\circ} \mathrm{C}$ for $2 \mathrm{~h}$. The liquid of each well was removed, and $100 \mu \mathrm{l}$ of biotin antibody (1X) was added to each well. The assay plate was incubated at $37^{\circ} \mathrm{C}$ for $1 \mathrm{~h}$ with a new adhesive strip covering. Washing buffer (provided in the ELISA kit) was used to wash each well three times following 

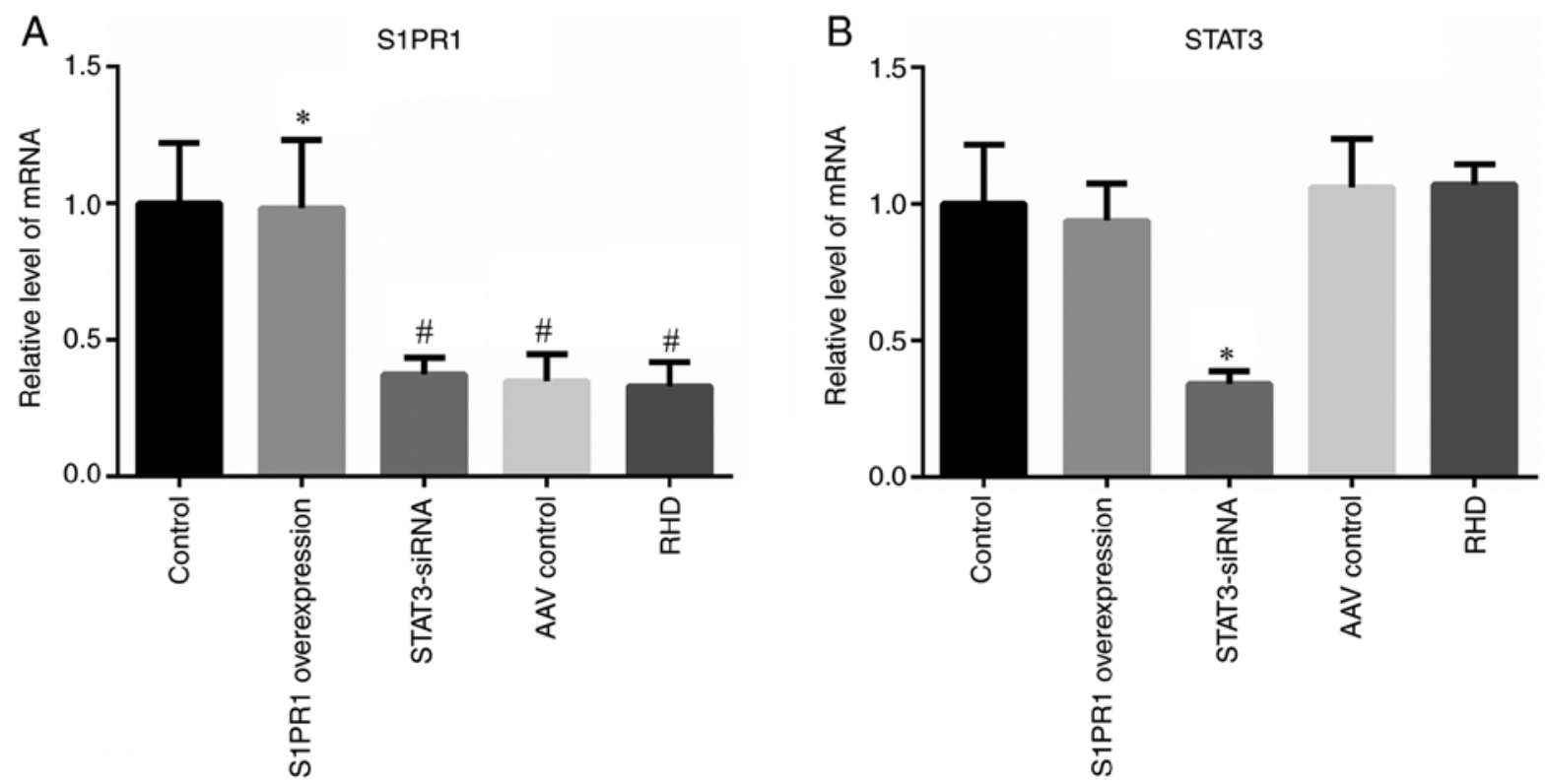

Figure 1. Reverse transcription-quantitative PCR. (A) Fold changes in the expression of S1PR1 mRNA between the 5 groups. (B) Fold changes in the expression of STAT3 mRNA between the 5 groups. These results indicated that S1PR1 mRNA was expressed at higher levels in the control group and S1PR1 overexpression group than the other 3 groups, and STAT3 mRNA was expressed at lower levels in the STAT3-siRNA group than the RHD group. Data are presented as the mean \pm standard deviation; ${ }^{\#} \mathrm{P}<0.05$ compared with the control group; ${ }^{*} \mathrm{P}<0.05$ compared with the RHD group. S1PR1, sphingosine-1-phosphate receptor 1; STAT3, signal transducer and activator of transcription 3; RHD, rheumatic heart disease.

the removal of the liquid from each well. HRP-avidin (1X, $100 \mu \mathrm{l})$ was added to each well, covered with a new adhesive strip, and incubated at $37^{\circ} \mathrm{C}$ for $1 \mathrm{~h}$. Each well was washed five times, $90 \mu 1 \mathrm{TMB}$ substrate were added to each well, and the assay plate was placed in the dark at $37^{\circ} \mathrm{C}$ for $15-30 \mathrm{~min}$. A stop solution $(50 \mu \mathrm{l})$ was added to each well to stop the reaction. The sample concentrations were calculated based on the absorbance of each well.

Statistical analysis. For data other than IHS, the results are presented as the means \pm standard deviations of at least three independent experiments. SPSS software 17.0 (SPSS, Inc.) was used for statistical analyses. One-way ANOVA was used to compare differences between the 5 groups with Tukey's test as the post hoc multiple comparisons test. For the data of IHS, the Kruskal-Wallis test was used, and Dunn's test was used as the post hoc test. The results are expressed as the median and interquartile range. $\mathrm{P}<0.05$ was considered to indicate a statistically significant difference.

\section{Results}

\section{Overexpression of SIPRI}

In vivo gene therapy increases SIPRI expression. The results of RT-qPCR, WB and immunohistochemistry revealed a significantly lower expression of S1PR1 in the AAV control and RHD groups than in the control group $(\mathrm{P}<0.05)$; these findings were consistent with those of previous studies $(14,26)$. The expression in the S1PR1 overexpression group was similar to that in the control group, and it was significantly higher than that in the RHD group ( $\mathrm{P}<0.05$; Figs. 1A, $2 \mathrm{~A}$ and $\mathrm{B}$, and $3 \mathrm{~A}-\mathrm{C})$. These results indicated that the S1PR1 overexpression sequence used for overexpression in the present study successfully increased the expression of S1PR1.
The level of phosphorylated STAT3 is reduced with S1PR1 overexpression. The results of WB, RT-qPCR and immunohistochemistry did not reveal significant differences in STAT3 expression between the control, S1PR1 overexpression, AAV control and RHD groups (Figs. 1B, 2A and C, and 3A-C). Significantly higher levels of p-STAT3 were detected in the AAV control and RHD groups than in the control group $(\mathrm{P}<0.05)$. Significantly lower levels of $\mathrm{p}$-STAT3 were detected in the S1PR1 overexpression group than in the RHD group ( $\mathrm{P}<0.05$; Figs. $2 \mathrm{~A}$ and $\mathrm{D}$, and $3 \mathrm{~A}-\mathrm{C})$. The ratio of p-STAT3/total (t-)STAT3 also exhibited a similar trend ( $\mathrm{P}<0.05$; Figs. 2E and 3D). Therefore, the level of phosphorylated STAT3 was reduced.

The expression of Th17 cell-related factors in the S1PRI overexpression group is significantly lower than that in the RHD group. Immunohistochemistry and ELISA were then performed to determine the levels of Th17 cell-related factors. The results revealed significantly higher levels of ROR $\gamma \mathrm{t}$, IL-6 and IL-17 in the AAV control and the RHD groups than in the control group $(\mathrm{P}<0.05)$. The S1PR1 overexpression group exhibited significantly lower levels of ROR $\gamma$ t, IL-6 and IL-17 than the RHD group ( $\mathrm{P}<0.05 ;$ Fig. 4). Thus, S1PR1 overexpression reduced the levels of Th17 cell-related factors in valvular tissue and serum.

Elevated SIPRI expression attenuates RHD-induced valvular damage. H\&E and Sirius Red staining revealed inflammation and fibrosis in the valvular tissue of the AAV control and RHD groups. The control group exhibited a normal valvular structure; however, the valves in the S1PR1 overexpression group exhibited reduced inflammation (Fig. 5A) and fibrosis (Fig. 5B) compared with those in the RHD group. Type 1 collagen (COL1) fibres are the main type of collagen in non-fibrotic valves (30). The ratio of type 3 collagen (COL3) and COL1 is used to reflect the degree of fibrosis in 
A

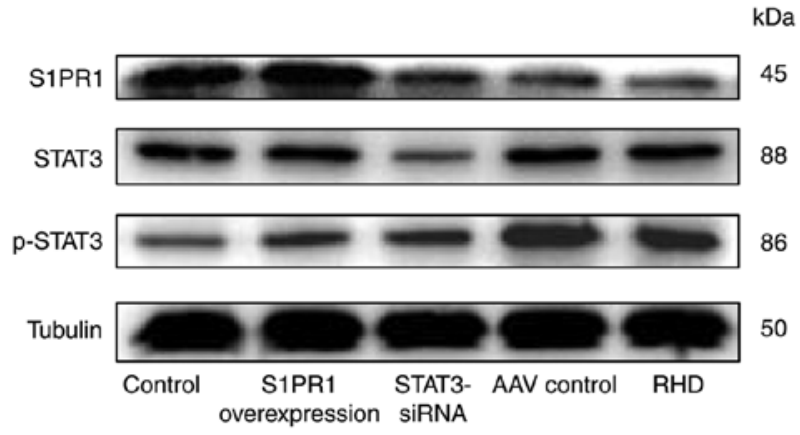

C

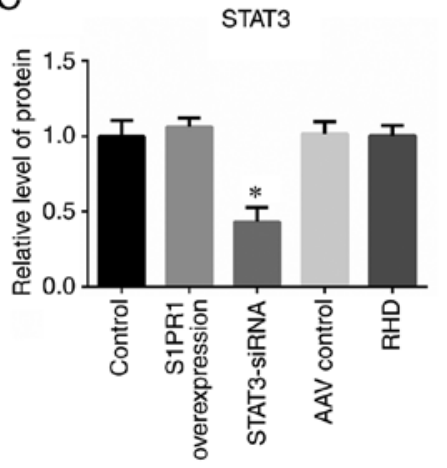

D

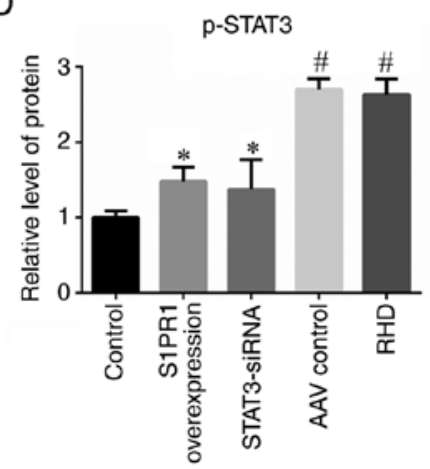

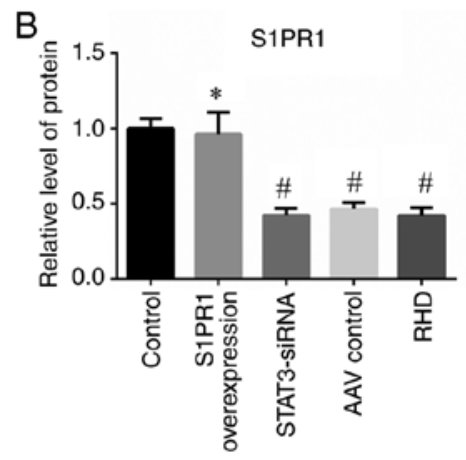

$\mathrm{E}$

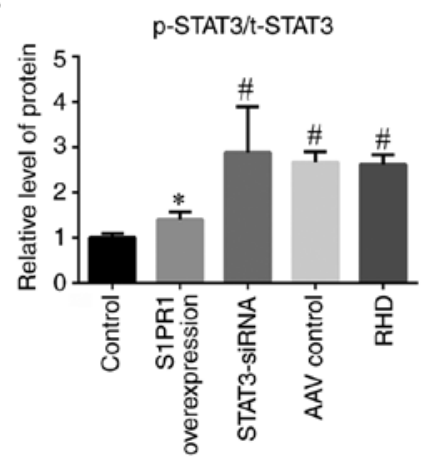

Figure 2. Western blot analysis. (A) Bands indicating the levels of the S1PR1, STAT3 and p-STAT3 proteins in the 5 groups. (B) Fold changes in the level of S1PR1 protein between the 5 groups. (C) Fold changes in the level of STAT3 protein between the 5 groups. (D) Fold changes in the level of p-STAT3 protein between the 5 groups. (E) Relative ratios of the levels of p-STAT3/t-STAT3 proteins. These results revealed that S1PR1 overexpression increased the protein levels of S1PR1, and the levels of p-STAT3 in the S1PR1 overexpression group were lower than those in the RHD group, and the silencing of STAT3 by STAT3-siRNA decreased the level of the STAT3 protein and the total amount of p-STAT3 in valve tissue. Data are presented as the mean \pm standard deviation; ${ }^{\#} \mathrm{P}<0.05$ compared with the control group; ${ }^{*} \mathrm{P}<0.05$ compared with the RHD group. S1PR1, sphingosine-1-phosphate receptor 1; STAT3, signal transducer and activator of transcription 3; RHD, rheumatic heart disease; t-STAT3, total-signal transducer and activator of transcription 3; p-STAT3, phosphorylated signal transducer and activator of transcription 3.

valve tissue. During the process of fibrosis of valvular tissue, the proportion of COL3 increases, indicating more severe fibrosis (30). Therefore, the present study calculated the COL3/COL1 (COL3/1) ratio to quantify the degree of fibrosis in valve tissue. The Sirius Red staining images revealed that COL1 fibres were closely packed yellow and red fibres with obvious birefringence, and COL 3 fibres were loosely arranged green fibres with weak birefringence (Fig. 5B). The ratio of COL3/COL1 in the S1PR1 overexpression group was significantly lower than that in the RHD group $(\mathrm{P}<0.05$; Fig. $5 \mathrm{C})$. The expression of COL3a1 and fibroblast-specific protein 1 (FSP1) was also detected by RT-qPCR to examine the degree of valve fibrosis at the mRNA level. COL3a1 and FSP1 were expressed at significantly lower levels in the S1PR1 overexpression group than the RHD group ( $\mathrm{P}<0.05$; Fig. 5D and $\mathrm{E})$. These results demonstrated that S1PR1 overexpression attenuated RHD-induced valvular damage.

\section{Inhibition of STAT3}

STAT3-siRNA pre-treatment decreases STAT3 expression and reduces the total amount of p-STAT3. The silencing of STAT3 using STAT3-siRNA decreased the expression of STAT3 mRNA (P<0.05; Fig. 1B). A significantly higher protein level of p-STAT3 was observed in the RHD and AAV control groups than in the control group $(\mathrm{P}<0.05)$. The silencing of STAT3 by STAT3-siRNA decreased the protein levels of STAT3 and p-STAT3 in valvular tissues $(\mathrm{P}<0.05$; Figs. $2 \mathrm{~A}, \mathrm{C}$ and D, and
3A-C). The ratio of p-STAT3/t-STAT3 was significantly higher in the STAT3-siRNA, AAV control and RHD groups than in the control group $(\mathrm{P}<0.05)$; however, a significant difference was not observed between the STAT3-siRNA, AAV control and RHD groups $(\mathrm{P}<0.05$; Figs. $2 \mathrm{E}$ and $3 \mathrm{D})$.

Expression of Th17-related transcription factors and cytokines is reduced in the STAT3-siRNA group compared with the RHD group. Immunohistochemistry and ELISA revealed significantly higher levels of ROR $\gamma \mathrm{t}$, IL-6 and IL-17 in the RHD and AAV-control groups than in the control group $(\mathrm{P}<0.05)$. The silencing of STAT3 by STAT3-siRNA decreased the levels of ROR $\gamma$ t, IL-6 and IL-17 in the serum and valvular tissues of the rats $(\mathrm{P}<0.05 ;$ Fig. 4$)$.

STAT3-siRNA pre-treatment attenuates RHD-induced valvular damage. H\&E staining revealed an inflammatory response in the heart valves of rats in the AAV control and RHD groups. The inflammatory response in the STAT3-siRNA group was reduced compared with that in the RHD group (Fig. 5A). All changes were observed under a microscope. The Sirius Red staining images revealed significantly more severe fibrosis in the RHD group than in the control group. The COL3/1 ratio was also significantly higher in the RHD group than in the control group. The degree of fibrosis in the STAT3-siRNA group was lower than the RHD group. The COL3/1 ratio was significantly lower in the STAT3-siRNA group than the RHD group $(\mathrm{P}<0.05$; Fig. $5 \mathrm{~B}$ and $\mathrm{C})$. The findings for the COL3a1 and FSP1 expression levels were 
A
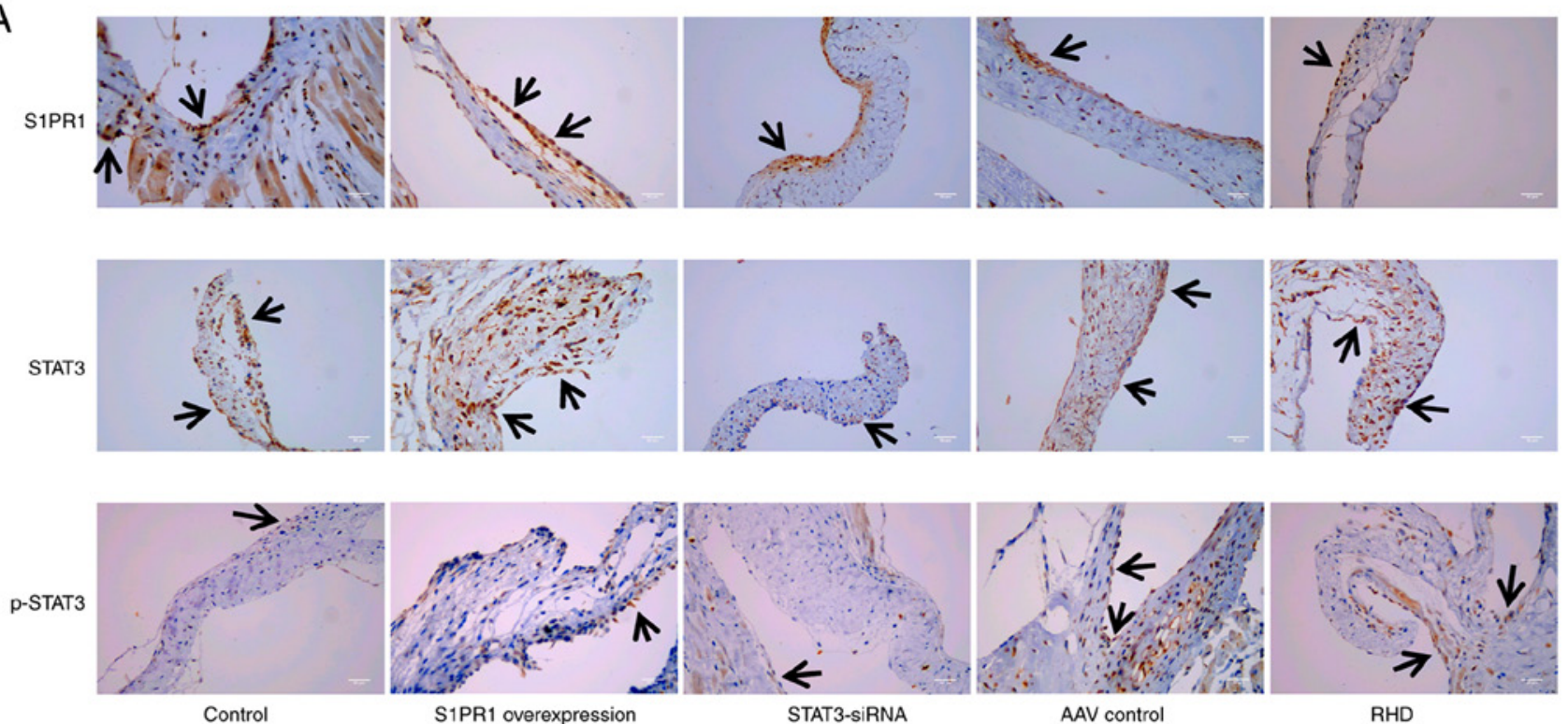

B
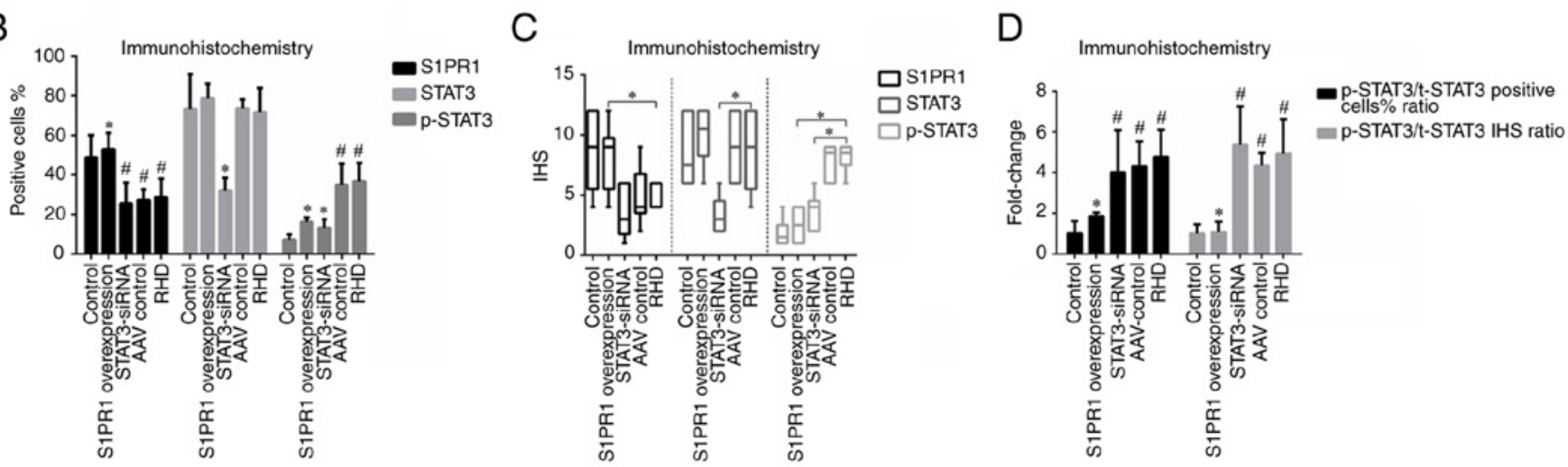

Figure 3. Immunohistochemical staining for S1PR1, STAT3 and p-STAT3. (A) Immunohistochemical staining for S1PR1, STAT3 and p-STAT3 in valvular tissues; magnification, x400; scale bar, $50 \mu \mathrm{m}$. Arrows indicate positively stained cells. (B) Percentage of positive cells. (C) The IHS. (D) Ratio of p-STAT3/t-STAT3. These results revealed that S1PR1 was expressed at higher levels in the control group and S1PR1 overexpression group than the other 3 groups, and the levels of p-STAT3 in the S1PR1 overexpression group were lower than those in the RHD group, and the silencing of STAT3 by STAT3-siRNA decreased the level of the STAT3 protein and the total amount of p-STAT3 in valvular tissue. Data are presented as the mean \pm standard deviation and the median and interquartile range; ${ }^{\text {"}} \mathrm{P}<0.05$ compared with the control group. ${ }^{*} \mathrm{P}<0.05$ compared with the RHD group. S1PR1, sphingosine-1-phosphate receptor 1; STAT3, signal transducer and activator of transcription 3; RHD, rheumatic heart disease; IHS, immunohistochemical score; t-STAT3, total-signal transducer and activator of transcription 3; p-STAT3, phosphorylated signal transducer and activator of transcription 3.

consistent with the results of the histological examination $(\mathrm{P}<0.05$; Fig. 5D and $\mathrm{E})$. These results demonstrated that the inflammatory response and fibrosis of the valvular tissue were reduced following the silencing of STAT3 compared with the RHD group.

\section{Discussion}

RHD has a long history, and a number of patients have succumbed to this disease. RHD caused 319,400 deaths in 2015 (31), 314,600 deaths in 2016 (32) and 285,500 deaths in 2017 (33); however, the pathogenesis of this disease remains unknown. Recent research has primarily focused on the signalling pathways related to the pathogenesis of RHD. The efforts of numerous researchers have elucidated some of the signalling pathways related to this disease. Recently, researchers have discovered that the S1PR1/STAT3 signalling pathway is involved in RHD-induced valvular damage in a rat model (14). However, the mechanisms through which interventions targeting the expression of S1PR1 and STAT3 affect RHD-induced valvular damage remain unknown. Notably, the most useful method which can be used to intervene with the expression of S1PR1 and STAT3, and effectively attenuate RHD-induced valvular damage is not yet clear.

S1PR1 has been extensively studied, and it is an important factor in heart diseases, including RHD $(14,26)$, myocardial infarction (9) and cardiac remodelling (10). S1PR1 primarily protects the heart in these diseases (9-11), and a high S1PR1 expression generally protects the heart during the pathogenesis of heart disease $(9,12)$. However, a previous study reported that a high expression of S1PR1 exacerbated heart damage (34), and the role of the downregulation of S1PR1 expression in mediating the pathogenesis of other diseases, such as multiple sclerosis, has also been reported (35). The low expression of S1PR1 is not only present in RHD. For example, studies have reported that in tumours, the low expression or lack of S1PR1 aggravates the growth of tumours, and the high expression of S1PR1 can enhance the antitumour ability of the body (36). 
A
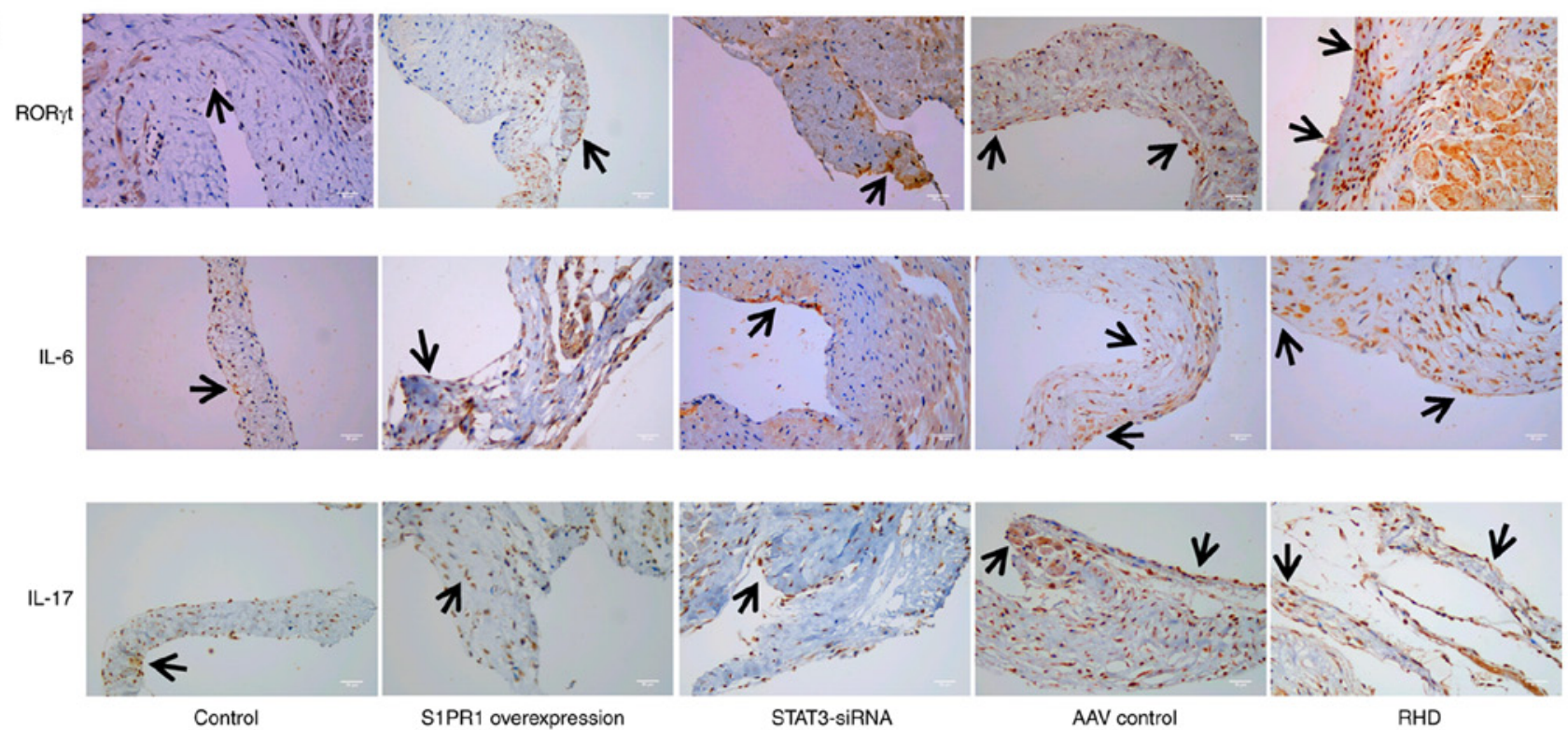

B

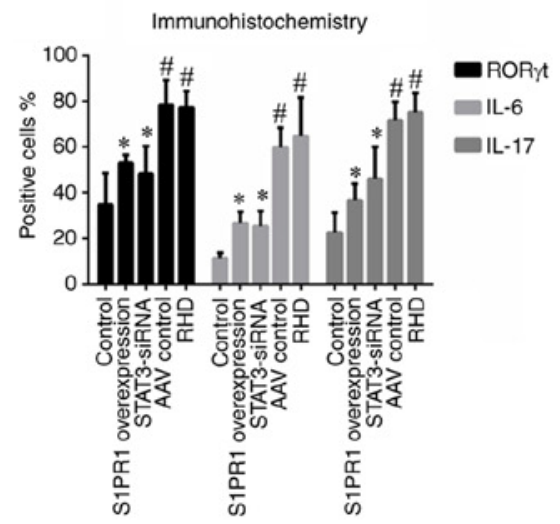

C

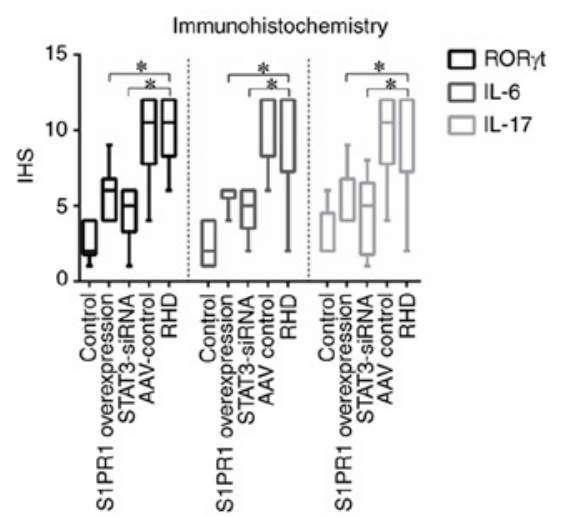

D

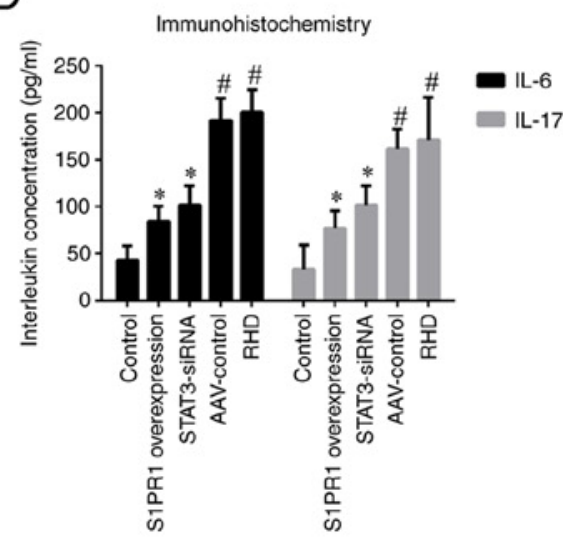

Figure 4. Levels of Th17 cell-related transcription factors and cytokines. (A) Immunohistochemical staining for ROR $\gamma$ t, IL-6 and IL-17 in valvular tissues; magnification, $\mathrm{x} 400$; scale bar, $50 \mu \mathrm{m}$. Arrows indicate positively stained cells. (B) Percentage of positive cells. (C) The IHS. (D) ELISA of serum IL-6 and IL-17 levels. These results revealed that the levels of ROR $\gamma$ t, IL-6 and IL-17 were reduced in the S1PR1 overexpression group and STAT3-siRNA group. Data are presented as the mean \pm standard deviation and the median and interquartile range. ${ }^{\#} \mathrm{P}<0.05$ compared with the control group; ${ }^{*} \mathrm{P}<0.05$ compared with the RHD group. ROR $\gamma$ t, retinoic acid-related orphan receptor $\gamma \mathrm{T}$; IL, interleukin; IHS, immunohistochemical score; RHD, rheumatic heart disease.

Studies on hypertension have also reported that the expression of S1PR1 is downregulated, and increasing the expression of S1PR1 is helpful for reducing hypertension (36). It may be related to RHD being an autoimmune disease. The low expression of S1PR1 has also been observed in autoimmune diseases (multiple sclerosis) and Crohn's disease (37). S1PR1 may also be regulated by microRNAs (miRNAs/miRs) to downregulate its expression (38), such as miR-155-5p (26). Therefore, it can be concluded that the expression of S1PR1 in different heart diseases is not static. Although S1PR1 expression varies among heart diseases, S1PR1 expression is generally upregulated and it exerts a cardioprotective effect. The present study found that RHD-induced valvular damage was reduced with S1PR1 overexpression, which may also be related to the cardioprotective effects of S1PR1. A close association was identified between S1PR1 and STAT3, and a number of studies have described the regulatory effect of S1PR1 on STAT3 in various diseases (22-25). The present study demonstrated that the expression of S1PR1 and STAT3 was closely related to the pathogenesis of valvular damage in a rat model of RHD. Previous studies on the process of valvular damage in RHD have found that the downregulation of S1PR1 and the increased STAT3 phosphorylation are involved in this process $(14,26)$. This phenomenon of a downregulated S1PR1 expression and an increased STAT3 phosphorylation has been reported in previous studies. For example, Garris et al (13) found that S1PR1 deficiency increased the level of p-STAT3 and promoted Th17 cell differentiation in a mouse autoimmune encephalitis model with an S1PR1 gene mutation. The present study demonstrated that the RHD group also exhibited a decreased S1PR1 expression and an increased STAT3 phosphorylation compared with that in the control group. However, an increase in S1PR1 expression has been reported to increase the phosphorylation of STAT3 $(23,39)$; however, these studies did not examine RHD. Combined with the uncertainty of the expression of S1PR1 in the different heart diseases mentioned above, it can be concluded that the expression of S1PR1 and its role in regulating the phosphorylation of STAT3 in different diseases and different physiological or pathological processes may not be static. However, the opposite trend of S1PR1 and 
A

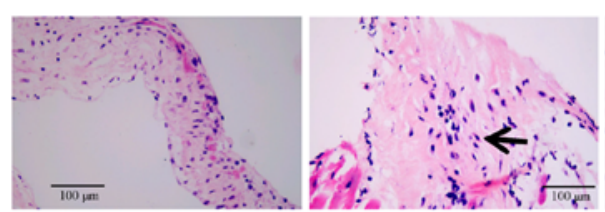

Control

B

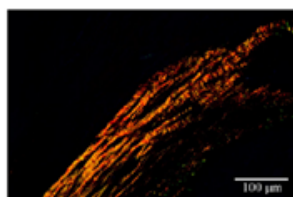

Control
S1PR1 overexpression

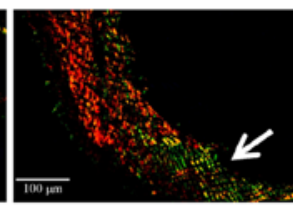

S1PR1 overexpression

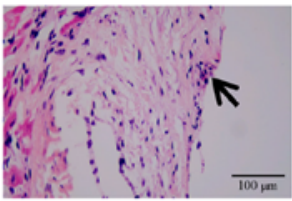

STAT3-SIRNA

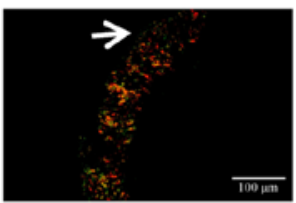

STAT3-siRNA

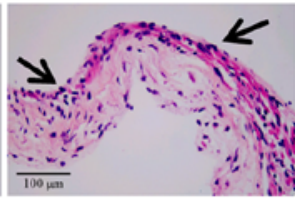

AAV control

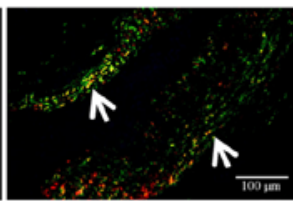

AAV control

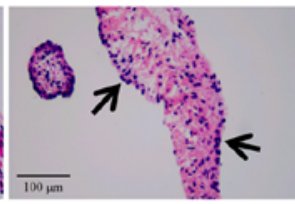

$\mathrm{RHD}$

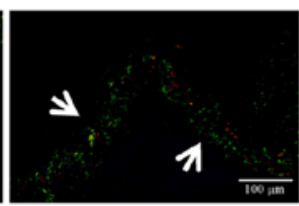

RHD
C

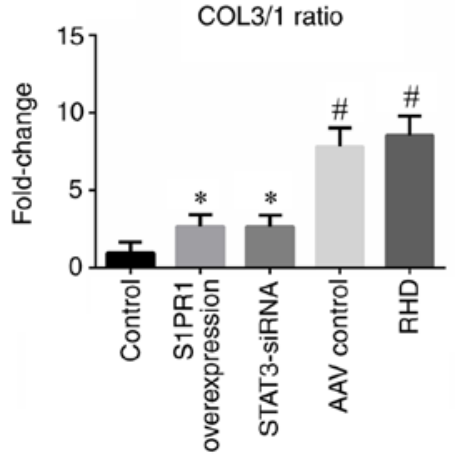

D

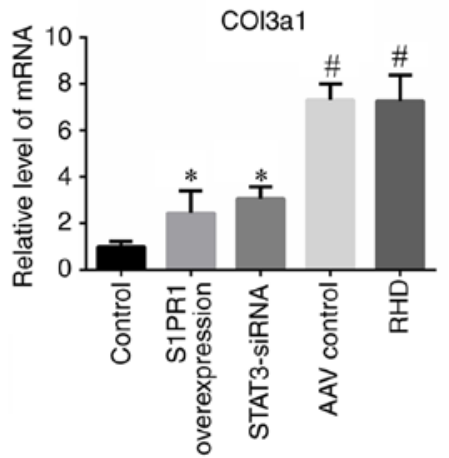

E

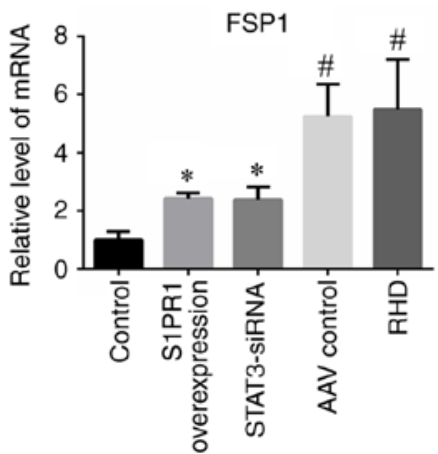

Figure 5. H\&E and Sirius Red staining of valvular tissues and RT-qPCR analysis of fibrosis-related factors. (A) H\&E staining revealed an inflammatory response in the heart valves of the rats in the AAV control and RHD groups. In the S1PR1 overexpression and STAT3-siRNA groups, the inflammatory response was significantly reduced compared with that in the RHD group; magnification, $\mathrm{x} 400$; scale bar, $100 \mu \mathrm{m}$. Arrows indicate the inflammatory response. (B) Images of Sirius red staining of the valves. The valves in the AAV control group and RHD group exhibited marked fibrosis. In the S1PR1 overexpression and STAT3-siRNA groups, the degree of fibrosis was markedly reduced compared with that in the RHD group; magnification, $\mathrm{x} 400$; scale bar, $100 \mu \mathrm{m}$. Arrows indicate COL3 expression. (C) A significantly higher COL3/1 ratio was observed in the RHD group than in the control group, and the COL3/1 ratio in the S1PR1 overexpression and STAT3-siRNA groups was significantly lower than that in the RHD group. (D and E) RT-qPCR analysis of COL3a1 and FSP1 expression. These results revealed that the degree of valvular damage was at significantly higher levels in the RHD group than the control group. S1PR1 overexpression or STAT3-silencing reduced the level of valvular damage. Data are presented as the mean \pm standard deviation. ${ }^{\#} \mathrm{P}<0.05$ compared with the control group; "P<0.05 compared with the RHD group. H\&E, Hematoxylin and eosin staining; RT-qPCR, reverse transcription-quantitative PCR; COL3, collagen fibre type 3; COL1, collagen fibre type 1; Col3a1, collagen type III $\alpha 1$ chain; FSP1, fibroblast-specific protein 1; RHD, rheumatic heart disease.

p-STAT3 also indicates the possibility that p-STAT3 is indirectly regulated by S1PR1. Two previous studies on the roles of S1PR1 and STAT3 in RHD $(14,26)$ and the experimental results presented in the present study demonstrated that the downregulation of S1PR1 and the upregulation of phosphorylation of STAT3 during RHD-induced valvular damage and induces the differentiation of Th17 cells. Therefore, the mechanism of the S1PR1 and STAT3 during the process of RHD-induced valvular damage may be very similar to the mechanism described by Garris et al (13), as autoimmune encephalitis and RHD are autoimmune diseases. However, the similarity of the mechanisms is purely a speculation. S1PR1 is downregulated in this pathway, and whether the overexpression of S1PR1 would attenuate RHD-induced valvular damage was not known.

STAT3 is a key pathogenic factor in many inflammatory conditions. STAT3 mediates immune myocarditis due to IL-6-induced liver complement component C3 production and Th17 cell differentiation (40), and the differentiation of Th17 cells plays an important role in the occurrence and development of myocarditis (41). Tissue signalling cytokines, such as IL-17 and IL-22, may affect the heart via a pathway that involves STAT3 (42). Th17 cells and related inflammatory factors (such as IL-17) play important roles in the process of inflammation and the autoimmune response (17-19). The levels of Th17 cell-related factors are increased in the peripheral blood and serum of patients with RHD (20), and the level of Th17-related cytokines in the mitral valve is significantly increased (21). Therefore, Th17 cells likely promote the development of RHD. Previous researchers have reported high levels of p-STAT3 in individuals with rheumatoid arthritis (43), and the present study considered STAT3 a key component of this signalling pathway. The present study wished to determine whether the suppression of STAT3 expression would attenuate RHD-induced valvular damage.

Based on studies on the association between the expression of S1PR1, STAT3 and RHD-induced valvular damage, it was hypothesised that the level of p-STAT3 was increased during the process of valvular damage, which promoted the differentiation of $\mathrm{CD}^{+} \mathrm{T}$ cells into Th17 cells and the Th17 cell-related cytokines to participate in the process of RHD-induced valvular damage. Therefore, experiments were performed to overexpress S1PR1 and inhibit STAT3. The results obtained from in the first part of the present study demonstrated that the originally downregulated expression of S1PR1 was increased with S1PR1 overexpression, the level of 
p-STAT3 was decreased, the levels of Th17 cell-related cytokines in the valvular tissue and serum were also decreased, and eventually, the level of RHD-induced inflammation and fibrosis of the valve was attenuated. In previous studies, it was also demonstrated that the overexpression of S1PR1 caused the expression of p-STAT3 to decrease, and there was also no significant difference in the amount of STAT3 between groups $(14,26)$. This may have occurred as the intervention of S1PR1 affected the activation of STAT3, and the activation of STAT3 was mainly manifested in the amount of p-STAT3; thus, the amount of STAT3 had no effect, as a number of previous studies on STAT3 have observed the same phenomenon (44-46).

The results obtained from the second part of the present study demonstrated decreased levels of total STAT3 and p-STAT3, the decreased expression of Th17-related transcription factors and cytokines, and the attenuation of the level of RHD-induced inflammation and fibrosis of the valve following STAT3 silencing. There is a close association between STAT3, IL-6 and IL-17, it has been suggested that the three factors are mutually reinforcing (47). For example, STAT3 can promote the production of the pro-inflammatory cytokine, IL-6, and forms a positive feedback loop to regulate IL-6 level (48). The pharmacological inhibition of STAT3 with JAK or STAT3 inhibitors, or the inhibition of STAT3 genetically with dominant negative STAT3 and short hairpin STAT3 has been shown to reduce the level of IL-6 (49). The activation of STAT3 promotes the differentiation of Th17 cells. IL-6 and IL-17 are Th17-related cytokines; thus, the inhibition of STAT3 expression will affect the expression of IL-6 and IL-17 (50,51). These results demonstrate that the expression of S1PR1 and STAT3 is involved in the regulation of Th17 cell-related cytokine levels during RHD-induced valvular damage, and strategies designed to interfere with the expression of S1PR1 and STAT3 may modulate the expression of Th17 cell-related cytokines, and may subsequently attenuate RHD-induced valvular damage.

Studies investigating the signalling pathways related to the pathogenesis of RHD are limited. Significant research progress was achieved in only six signalling pathways: The RhoA/Rho-dependent kinase (RhoA/ROCK) signalling pathway, mitogen-activated protein kinase (MAPK) signalling pathway, protein kinase B/S6 kinase (AKT/S6K) signalling pathway, TGF- $\beta 1 /$ Smad signalling pathway, Wnt signalling pathway and S1PR1/STAT3 signalling pathway (52-57). Only three potential intervention targets in these signalling pathways were identified: The modulation of the expression of interferon (IFN) $-\gamma$ and tumour necrosis factor (TNF)- $\alpha$ to regulate extracellular matrix remodelling and reduce RHD-induced heart damage, altering the activity of the AKT/S6K signalling pathway to inhibit TGF- $\beta 1$-induced fibroblasts, and targeting the S1PR1/STAT3 signalling pathway to reduce RHD-induced valvular damage. Further studies are required to determine whether these intervention targets effectively prevent and treat RHD. International research on the pathogenesis of RHD is lacking, and the pathogenesis of RHD remains unclear. The threat to the lives and health of patients with RHD is substantial, and damage to the health and quality of life of patients is devastating. RHD is a severe condition, and studies investigating its pathogenesis are worthwhile. S1PR1 and STAT3 may prove to be two potential intervention targets for RHD.
The findings of the present study may enhance the current understanding of the signalling pathways related to the pathogenesis of RHD, thus contributing to the further understanding of the pathogenesis of RHD. The findings presented herein may also aid in the development of effective and inexpensive methods for controlling RHD in the future.

The present study has some limitations. The present study was performed using a rat model, and further studies using human samples are required. S1PR1 expression is downregulated during RHD-induced valvular damage, and greater technical requirements and further experiments are required to examine the effects of a complete inhibition of S1PR1 expression. The effects of upregulating STAT3 expression on RHD are unknown. Cell-based experiments may provide cell-level evidence to support the findings of the present study; however, such experiments were not performed herein. In addition, the present study did not detect the protein levels of FSP1 and COL3a1. The absence of data evaluating cardiac function in the RHD model following the overexpression of S1PR1/knockdown of STAT3 is also a potential limitation of the present study. The effect of other STAT3 inhibitors in the RHD model may be a good direction for further studies. The specific regulatory mechanisms between S1PR1 and STAT3, and whether there are other regulatory mechanisms between these two proteins warrants further investigation in the future.

The status of RHD remains severe, and although primary and secondary prevention strategies have been clearly identified, their global implementation is not ideal (58). The pathogenesis of RHD has long been studied (2); however, this is still not fully understood. By summarizing the results of previous studies and the inflammatory mechanisms of RHD pathogenesis, it was hypothesised that strategies targeting the expression of S1PR1 and STAT3 may modulate the process of RHD-induced valvular damage. In a previous study by the authors, it was found that S1PR1 and STAT3 may be involved in RHD (14); however, the specific roles of S1PR1 and STAT3 remain unclear. The animal experiments in the present study revealed a role for the expression of S1PR1 and STAT3 in regulating the levels of Th17 cell-related cytokines during RHD-induced valvular damage, and interfering with the expression of S1PR1 and STAT3 may alter the expression of Th17 cell-related cytokines and attenuate RHD-induced valvular damage. The present study provides some insight into the pathogenesis of RHD, and provides some references for discovering intervention targets for RHD. However, the present study found that only intervention with the expression of S1PR1 and STAT3 reduced RHD-induced valve damage. The specific regulatory mechanisms between S1PR1 and STAT3, whether there are other regulatory mechanisms between these two proteins, and whether this strategy is effective in in vitro experiments and in human samples needs to be further studied in the future.

\section{Acknowledgements}

Not applicable.

\section{Funding}

This study was supported by the National Natural Science Foundation of China (grant no. 81660069), the Guangxi Key 
Laboratory Base of Precision Medicine in Cardio-cerebrovascular Disease Control and Prevention (grant no. 17-259-85), the Guangxi Clinical Research Center for Cardio-cerebrovascular Diseases (grant no. AD17129014) and the Guangxi Medical High-level Backbone Talents ‘139’ Program (grant no. G201901006).

\section{Availability of data and materials}

All data generated or analysed during this study are included in this published article.

\section{Authors' contributions}

$\mathrm{ZZ}$ and FH conceived and designed the study. SX and AC participated in the experimental design. SX, AC, YW and CL performed the experiments. SX, AC and HW analysed the data. SX and FH confirm the authenticity of all the raw data. SX wrote the manuscript, and all authors reviewed, and have read and approved the final manuscript.

\section{Ethics approval and consent to participate}

Protocols involving animals were approved by The Medical Ethics Committee of the First Affiliated Hospital of Guangxi Medical University (Nanning, China; approval no. 2019-KY-E-069).

\section{Patient consent for publication}

Not applicable.

\section{Competing interests}

The authors declare that they have no competing interests.

\section{References}

1. Watkins DA, Johnson CO, Colquhoun SM, Karthikeyan G, Beaton A, Bukhman G, Forouzanfar MH, Longenecker CT, Mayosi BM, Mensah GA, et al: Global, regional, and national burden of rheumatic heart disease, 1990-2015. N Engl J Med 377: 713-722, 2017.

2. Leal MT, Passos LS, Guarçoni FV, Aguiar JM, Silva RB, Paula TM, Santos RF, Nassif MC, Gomes NF, Tan TC and Nunes MC: Rheumatic heart disease in the modern era: Recent developments and current challenges. Rev Soc Bras Med Trop 52: e20180041, 2019.

3. Mirabel M, Narayanan K, Jouven X and Marijon E: Cardiology patient page. Prevention of acute rheumatic fever and rheumatic heart disease. Circulation 130: e35-e37, 2014.

4. Zhao Z, He D, Ling F, Chu T, Huang D, Wu H and Ge J: CD4 $\mathrm{T}$ cells and TGF $\beta 1 /$ MAPK signal pathway involved in the valvular hyperblastosis and fibrosis in patients with rheumatic heart disease. Exp Mol Pathol 114: 104402, 2020.

5. Messias-Reason IJ, Schafranski MD, Kremsner PG and Kun JF: Ficolin 2 (FCN2) functional polymorphisms and the risk of rheumatic fever and rheumatic heart disease. Clin Exp Immunol 157: 395-399, 2009.

6. Li M, Xu S, Geng Y, Sun L, Wang R, Yan Y, Wang H, Li Y, Yi Q, Zhang Y, et al: The protective effects of L-carnitine on myocardial ischaemia-reperfusion injury in patients with rheumatic valvular heart disease undergoing CPB surgery are associated with the suppression of NF- $\mathrm{BB}$ pathway and the activation of Nrf2 pathway. Clin Exp Pharmacol Physiol 46: 1001-1012, 2019.

7. Aoki M, Aoki H, Ramanathan R, Hait NC and Takabe K: Sphingosine-1-phosphate signaling in immune cells and inflammation: Roles and therapeutic potential Mediators Inflamm 2016: 8606878,2016
8. Jozefczuk E, Guzik TJ and Siedlinski M: Significance of sphingosine-1-phosphate in cardiovascular physiology and pathology. Pharmacol Res 156: 104793, 2020.

9. Deng S, Zhou X, Ge Z, Song Y, Wang H, Liu X and Zhang D: Exosomes from adipose-derived mesenchymal stem cells ameliorate cardiac damage after myocardial infarction by activating S1P/SK1/S1PR1 signaling and promoting macrophage M2 polarization. Int J Biochem Cell Biol 114: 105564, 2019.

10. Liu X, Wu J, Zhu C, Liu J, Chen X, Zhuang T, Kuang Y, Wang Y, $\mathrm{Hu} \mathrm{H}$, Yu P, et al: Endothelial S1prl regulates pressure overload-induced cardiac remodelling through AKT-eNOS pathway. J Cell Mol Med 24: 2013-2026, 2020.

11. Liu Y, Zhi Y, Song H, Zong M, Yi J, Mao G, Chen L and Huang G: S1PR1 promotes proliferation and inhibits apoptosis of esophageal squamous cell carcinoma through activating STAT3 pathway. J Exp Clin Cancer Res 38: 369, 2019.

12. Chen YZ, Wang F, Wang HJ and Liu HB: Sphingosine 1 phosphate receptor-1 (S1PR1) signaling protects cardiac function by inhibiting cardiomyocyte autophagy. J Geriatr Cardiol 15: 334-345, 2018.

13. Garris CS, Wu L, Acharya S, Arac A, Blaho VA, Huang Y, Moon BS, Axtell RC, Ho PP, Steinberg GK, et al: Defective sphingosine 1-phosphate receptor 1 (S1P1) phosphorylation exacerbates TH17-mediated autoimmune neuroinflammation. Nat Immunol 14: 1166-1172, 2013.

14. Wu XD, Zeng ZY, Gong DP, Wen JL and Huang F: Potential involvement of S1PR1/STAT3 signaling pathway in cardiac valve damage due to rheumatic heart disease. Biotech Histochem 94: 398-403, 2019.

15. Hu YS, Han X and Liu XH: STAT3: A potential drug target for tumor and inflammation. Curr Top Med Chem 19: 1305-1317, 2019.

16. Liu X, Hu H, Fan H, Zuo D, Shou Z, Liao Y, Nan Z and Tang Q: The role of STAT3 and AhR in the differentiation of $\mathrm{CD}^{+} \mathrm{T}$ cells into Th17 and Treg cells. Medicine (Baltimore) 96: e6615, 2017.

17. Gaffen SL, Jain R, Garg AV and Cua DJ: The IL-23-IL-17 immune axis: From mechanisms to therapeutic testing. Nat Rev Immunol 14: 585-600, 2014.

18. Whibley N, Tritto E, Traggiai E, Kolbinger F, Moulin P, Brees D, Coleman BM, Mamo AJ, Garg AV, Jaycox JR, et al: Antibody blockade of IL-17 family cytokines in immunity to acute murine oral mucosal candidiasis. J Leukoc Biol 99: 1153-1164, 2016.

19. Zhang Y, Shao Z, Zhang X, Jia X, Xia Y, Zhang Y, Xin N, Guo M, Chen J, Zheng S, et al: TIPE2 play a negative role in TLR4-mediated autoimmune T helper 17 cell responses in patients with myasthenia gravis. J Neuroimmune Pharmacol 10: 635-644, 2015.

20. Bas HD, Baser K, Yavuz E, Bolayir HA, Yaman B, Unlu S, Cengel A, Bagriacik EU and Yalcin R: A shift in the balance of regulatory $\mathrm{T}$ and $\mathrm{T}$ helper 17 cells in rheumatic heart disease. J Investig Med 62: 78-83, 2014.

21. Wen Y, Zeng Z, Gui C, Li L and Li W: Changes in the expression of Th17 cell-associated cytokines in the development of rheumatic heart disease. Cardiovasc Pathol 24: 382-387, 2015.

22. Lankadasari MB, Aparna JS, Mohammed S, James S, Aoki K, Binu VS, Nair S and Harikumar KB: Targeting S1PR1/STAT3 loop abrogates desmoplasia and chemosensitizes pancreatic cancer to gemcitabine. Theranostics 8: 3824-3840, 2018.

23. Lin Q, Ren L, Jian M, Xu P, Li J, Zheng P, Feng Q, Yang L, Ji M, Wei Y and Xu J: The mechanism of the premetastatic niche facilitating colorectal cancer liver metastasis generated from myeloid-derived suppressor cells induced by the S1PR1-STAT3 signaling pathway. Cell Death Dis 10: 693, 2019.

24. Lee H, Deng J, Kujawski M, Yang C, Liu Y, Herrmann A, Kortylewski M, Horne D, Somlo G, Forman S, et al: STAT3-induced S1PR1 expression is crucial for persistent STAT3 activation in tumors. Nat Med 16: 1421-1428, 2010.

25. Deng J, Liu Y, Lee H, Herrmann A, Zhang W, Zhang C, Shen S, Priceman SJ, Kujawski M, Pal SK, et al: S1PR1-STAT3 signaling is crucial for myeloid cell colonization at future metastatic sites. Cancer Cell 21: 642-654, 2012.

26. Chen A, Wen J, Lu C, Lin B, Xian S, Huang F, Wu Y and Zeng Z: Inhibition of miR-155-5p attenuates the valvular damage induced by rheumatic heart disease. Int J Mol Med 45: 429-440, 2020.

27. Livak KJ and Schmittgen TD: Analysis of relative gene expression data using real-time quantitative PCR and the 2(-Delta Delta C(T)) method. Methods 25: 402-408, 2001.

28. Guendisch U, Weiss J, Ecoeur F, Riker JC, Kaupmann K, Kallen J, Hintermann S, Orain D, Dawson J, Billich A and Guntermann C: Pharmacological inhibition of ROR $\gamma \mathrm{t}$ suppresses the Th17 pathway and alleviates arthritis in vivo. PLoS One 12: e0188391, 2017. 
29. Friedrichs K, Gluba S, Eidtmann $\mathrm{H}$ and Jonat W: Overexpression of p53 and prognosis in breast cancer. Cancer 72: 3641-3647, 1993.

30. Purushothaman KR, Purushothaman M, Turnbull IC, Adams DH, Anyanwu A, Krishnan P, Kini A, Sharma SK, O'Connor WN and Moreno PR: Association of altered collagen content and lysyl oxidase expression in degenerative mitral valve disease. Cardiovasc Pathol 29: 11-18, 2017.

31. GBD 2015 Mortality and Causes of Death Collaborators: Global, regional, and national life expectancy, all-cause mortality, and cause-specific mortality for 249 causes of death, 1980-2015: A systematic analysis for the global burden of disease study 2015 Lancet 388: 1459-1544, 2016.

32. GBD 2016 Causes of Death Collaborators: Global, regional, and national age-sex specific mortality for 264 causes of death, 1980-2016: A systematic analysis for the global burden of disease study 2016. Lancet 390: 1151-1210, 2017.

33. GBD 2017 Causes of Death Collaborators: Global, regional, and national age-sex-specific mortality for 282 causes of death in 195 countries and territories, 1980-2017: A systematic analysis for the global burden of disease study 2017. Lancet 392: 1736-1788, 2018.

34. Zhang F, Xia Y, Yan W, Zhang H, Zhou F, Zhao S, Wang W, Zhu D, Xin C, Lee Y, et al: Sphingosine 1-phosphate signaling contributes to cardiac inflammation, dysfunction, and remodeling following myocardial infarction. Am J Physiol Heart Circ Physiol 310: H250-H261, 2016.

35. Edmonds Y, Milstien S and Spiegel S: Development of small-molecule inhibitors of sphingosine-1-phosphate signaling. Pharmacol Ther 132: 352-360, 2011

36. Cartier A, Leigh T, Liu CH and Hla T: Endothelial sphingosine 1-phosphate receptors promote vascular normalization and antitumor therapy. Proc Natl Acad Sci USA 117: 3157-3166, 2020.

37. Abarca-Zabalía J, García MI, Lozano Ros A, Marín-Jiménez I, Martínez-Ginés ML, López-Cauce B, Martín-Barbero ML, Salvador-Martín S, Sanjurjo-Saez M, García-Domínguez JM and López Fernández LA: Differential expression of SMAD genes and S1PR1 on circulating CD $4^{+} \mathrm{T}$ cells in multiple sclerosis and Crohn's disease. Int J Mol Sci 21: 676, 2020.

38. Zhang C, Shen J, Kong S, Zhang M, Zhang Q, Zhou J, Zhen X, Kang N, Jiang Y, Ding L, et al: MicroRNA-181a promotes follicular granulosa cell apoptosis via sphingosine-1-phosphate receptor 1 expression downregulation $\dagger$. Biol Reprod 101: 975-985, 2019.

39. Silva VR, Micheletti TO, Katashima CK, Lenhare L, Morari J, Moura-Assis A, de Lima-Júnior JC, Camargo JA, Passos GR, Gaspar RS, et al: Exercise activates the hypothalamic S1PR1-STAT3 axis through the central action of interleukin 6 in mice. J Cell Physiol 233: 9426-9436, 2018.

40. Camporeale A, Marino F, Papageorgiou A, Carai P, Fornero S Fletcher S, Page BD, Gunning P, Forni M, Chiarle R, et al: STAT3 activity is necessary and sufficient for the development of immune-mediated myocarditis in mice and promotes progression to dilated cardiomyopathy. EMBO Mol Med 5: 572-590, 2013.

41. Yuan J, Yu M, Lin QW, Cao AL, Yu X, Dong JH, Wang JP Zhang JH, Wang M, Guo HP, et al: Th17 cells contribute to viral replication in coxsackievirus B3-induced acute viral myocarditis. J Immunol 185: 4004-4010, 2010.

42. Kurdi M, Zgheib C and Booz GW: Recent developments on the crosstalk Between STAT3 and inflammation in heart function and disease. Front Immunol 9: 3029, 2018.

43. Turkson J and Jove R: STAT proteins: Novel molecular targets for cancer drug discovery. Oncogene 19: 6613-6626, 2000.

44. Yang Y, Wang Y, Che X, Hou K, Wu J, Zheng C, Cheng Y, Liu Y, Hu X and Zhang J: Integrin $\alpha 5$ promotes migration and invasion through the FAK/STAT3/AKT signaling pathway in icotinib-resistant non-small cell lung cancer cells. Oncol Lett 22: $556,2021$.
45. Hu X, Jiao F, Zhang L and Jiang Y: Dihydrotanshinone Inhibits hepatocellular carcinoma by suppressing the JAK2/STAT3 pathway. Front Pharmacol 12: 654986, 2021.

46. Liu X, Zhou F, Wang W, Chen G, Zhang Q, Lv R, Zhao Z, Li X, Yu Q, Meves JM, et al: IL-9-triggered IncRNA Gm13568 regulates Notch1 in astrocytes through interaction with CBP/P300: Contribute to the pathogenesis of experimental autoimmune encephalomyelitis. J Neuroinflammation 18: 108, 2021.

47. Camporeale A and Poli V: IL-6, IL-17 and STAT3: A holy trinity in auto-immunity? Front Biosci (Landmark Ed) 17: 2306-2326, 2012.

48. Wu J, Niu P, Zhao Y, Cheng Y, Chen W, Lin L, Lu J, Cheng $\mathrm{X}$ and $\mathrm{Xu} \mathrm{Z}$ : Impact of miR-223-3p and miR-2909 on inflammatory factors IL-6, IL-1 $\beta$, and TNF- $\alpha$, and the TLR4/TLR2/NF- $\kappa$ B/STAT3 signaling pathway induced by lipopolysaccharide in human adipose stem cells. PLoS One 14 e0212063, 2019.

49. Bonetto A, Aydogdu T, Jin X, Zhang Z, Zhan R, Puzis L, Koniaris LG and Zimmers TA: JAK/STAT3 pathway inhibition blocks skeletal muscle wasting downstream of IL-6 and in experimental cancer cachexia. Am J Physiol Endocrinol Metab 303: E410-E421, 2012.

50. Damasceno LE, Prado DS, Veras FP, Fonseca MM, Toller-Kawahisa JE, Rosa MH, Públio GA, Martins TV, Ramalho FS, Waisman A, et al: PKM2 promotes Th17 cell differentiation and autoimmune inflammation by fine-tuning STAT3 activation. J Exp Med 217: e20190613, 2020.

51. Shui X, Chen S, Lin J, Kong J, Zhou C and Wu J: Knockdown of lncRNA NEAT1 inhibits Th17/CD4 ${ }^{+} \mathrm{T}$ cell differentiation through reducing the STAT3 protein level. J Cell Physiol 234: 22477-22484, 2019.

52. Jiang L, Wei XF, Yi DH, Xu P, Liu H, Chang Q, Yang SM, Li ZF, Gao HB and Hao GJ: Synergistic effects of cyclic strain and Th1-like cytokines on tenascin-C production by rheumatic aortic valve interstitial cells. Clin Exp Immunol 155: 216-223, 2009.

53. Li M, Yi XIN, Ma L and Zhou Y: Hepatocyte growth factor and basic fibroblast growth factor regulate atrial fibrosis in patients with atrial fibrillation and rheumatic heart disease via the mitogen-activated protein kinase signaling pathway. Exp Ther Med 6: 1121-1126, 2013

54. Zhang P, Wang W, Wang X, Wang X, Song Y, Zhang J and Zhao H: Focal adhesion kinase mediates atrial fibrosis via the AKT/S6K signaling pathway in chronic atrial fibrillation patients with rheumatic mitral valve disease. Int J Cardiol 168: 3200-3207, 2013.

55. Zhang L, Zhang N, Tang X, Liu F, Luo S and Xiao H: Increased $\alpha$-actinin-2 expression in the atrial myocardium of patients with atrial fibrillation related to rheumatic heart disease. Cardiology 135: 151-159, 2016.

56. Guo F, Yi X, Li M, Fu J and Li S: Snaill is positively correlated with atrial fibrosis in patients with atrial fibrillation and rheumatic heart disease. Exp Ther Med 14: 4231-4237, 2017.

57. Wu Y, Xu M, Bao H and Zhang JH: Sitagliptin inhibits EndMT in vitro and improves cardiac function of diabetic rats through the SDF-1 $\alpha /$ PKA pathway. Eur Rev Med Pharmacol Sci 23: 841-848, 2019.

58. Remenyi B, ElGuindy A, Smith SC Jr, Yacoub $M$ and Holmes DR Jr: Valvular aspects of rheumatic heart disease. Lancet 387: 1335-1346, 2016.

This work is licensed under a Creative Commons Attribution-NonCommercial-NoDerivatives 4.0 International (CC BY-NC-ND 4.0) License. 